Annuaire du Collège de France 2017-2018

\title{
Jacques Gernet (1921-2018)
}

\section{Anne Cheng}

\section{OpenEdition}

\section{Journals}

Édition électronique

URL : https://journals.openedition.org/annuaire-cdf/16624

DOI : 10.4000/annuaire-cdf.16624

ISBN : 978-2-7226-0572-5

ISSN : 2109-9227

Éditeur

Collège de France

Édition imprimée

Date de publication : 30 décembre 2020

Pagination : 731-733

ISBN : 978-2-7226-0516-9

ISSN : 0069-5580

\section{Référence électronique}

Anne Cheng, « Jacques Gernet (1921-2018)», L'annuaire du Collège de France [En ligne], 118 | 2020, mis en ligne le 01 avril 2021, consulté le 22 août 2022. URL : http://journals.openedition.org/annuaire-cdf/ 16624 ; DOI : https://doi.org/10.4000/annuaire-cdf.16624 


\section{HOMMAGES}

\section{JACQUES GERNET (1921-2018)}

Avec la disparition, le 3 mars dernier, de Jacques Gernet à l'âge de 96 ans, la sinologie française a perdu l'un de ses représentants les plus éminents. On peut même dire sans exagération aucune qu'il fut l'un des derniers géants d'une discipline représentée pour la toute première fois au plan institutionnel européen au Collège de France dès 1814, année où fut créée une chaire Langue et littérature tartaresmandchoues attribuée à Jean-Pierre Abel-Rémusat. Depuis lors, les études chinoises ont été représentées de manière quasi continue dans notre vénérable institution par de grands noms tels qu'Édouard Chavannes (1865-1918), Paul Pelliot (1878-1945) ou encore Henri Maspero (1883-1945), mort en déportation à Buchenwald en 1945. Le flambeau fut repris après la guerre par Paul Demiéville (1894-1969), titulaire de la chaire Langue et littérature chinoises de 1946 à 1964, que Jacques Gernet considérait comme son maitre et auquel il vouait une admiration sans borne.

Pour autant, Jacques Gernet était lui-même un géant par l'étendue de ses connaissances d'historien, reflétées en particulier dans son magnum opus traduit dans de nombreuses langues et mondialement connu, Le Monde chinois, connaissances qui allaient de la Chine ancienne d'avant l'ère chrétienne jusqu'à la Chine pré-moderne des XVII ${ }^{\mathrm{e}}-\mathrm{XVIII}{ }^{\mathrm{e}}$ siècles, ainsi que par la diversité de ses centres d'intérêt qui avaient trait à la pensée politique et philosophique de grandes figures telles que Wang Fuzhi (1619-1692), mais aussi aux aspects économiques du bouddhisme, à la perception de l'arrivée du christianisme par les lettrés chinois, aux rapports entre langue et pensée, sans oublier l'histoire des sciences.

Né le 22 décembre 1921 à Alger, Jacques Gernet était le fils du non moins éminent helléniste Louis Gernet (1882-1962), élève de Durkheim, ami de Granet et de Mauss, et historien du droit grec, qui venait d'être recruté à la jeune faculté des lettres d'Alger où il devait enseigner pendant près de trente ans, sa thèse de 1917 sur le développement de la pensée juridique et morale en Grèce, première pépite de l'anthropologie historique naissante, étant restée incomprise et lui ayant valu cette nomination que son disciple Jean-Pierre Vernant qualifiera de «relégation». Le jeune Gernet fut élève au lycée Bugeaud et commença par suivre la voie paternelle des études gréco-latines en obtenant une licence de lettres classiques et un diplôme d'études supérieures à Alger où son père était devenu doyen. Mais il dut interrompre ses études lorsqu'il fut mobilisé en 1942 à Blida. De sa jeunesse en Algérie et de ses 
années sous les drapeaux, Jacques Gernet, réservé et pudique par nature, ne parlait guère, pas plus qu'il n'évoquait le Manifeste des 121, déclaration sur le droit d'insoumission dans la guerre d'Algérie, qu'il signa courageusement avec son père en 1960. Du reste, malgré tous les honneurs et les distinctions qui lui furent décernés tout au long de sa vie, il ne portait pas ses décorations.

Son échec de jeunesse à l'agrégation de lettres classiques, qu'il devait ressasser toute sa vie, eut toutefois le mérite de l'orienter vers les études chinoises - une chance pour nous qui venons après lui et qui avons pu ainsi profiter de son immense érudition. Mais Jacques Gernet ne devait jamais oublier la leçon de son père dont il contribua à introduire l'approche en anthropologie historique dans le champ sinologique.

Durant les trente ans qui ont suivi la guerre, sa carrière universitaire l'aura fait passer par tout ce que les institutions françaises comptent en études extrêmeorientales. Rendu à la vie civile en 1945, il se lance dans l'apprentissage du chinois à Paris. Diplômé deux ans plus tard de l'École nationale des langues orientales vivantes (aujourd'hui l'Inalco), puis, l'année suivante, de l'École pratique des hautes études (EPHE), il intègre l'École française d'Extrême-Orient (EFEO), résidant de février 1949 à novembre 1950 à Hanoï où il publie sa première contribution scientifique majeure, la présentation et la traduction des enseignements de Chenhouei (Shenhui), patriarche du VIII ${ }^{\mathrm{e}}$ siècle de l'école bouddhiste du Chan (mieux connu sous le nom japonais de Zen).

À son retour en France, Jacques Gernet devient chercheur au CNRS, avant de se voir confier en 1955 une direction d'études à la VI ${ }^{\mathrm{e}}$ section de l'EPHE, appelée à devenir l'EHESS vingt ans plus tard. Sa thèse sur les aspects économiques du bouddhisme dans la société chinoise $\mathrm{du}^{\mathrm{e}}$ au $\mathrm{X}^{\mathrm{e}}$ siècle (qui soixante ans plus tard fait toujours figure d'œuvre pionnière) une fois soutenue en 1956, il devient l'année suivante maître de conférences, puis professeur, dès 1959, de langue et civilisation chinoise à la Sorbonne, poursuivant sa carrière dans le même poste, entre 1969 et 1973, à l'université Paris 7 dès sa création après la vague de Mai 68.

Rolf Stein (1911-1999), qui occupa au Collège de France de 1966 à 1981 la chaire Étude du monde chinois : institutions et concepts, fut celui qui plaida en 1974 pour l'élection de Jacques Gernet, présenté comme étant «le seul sinologue français qui s'est effectivement penché sur tous les aspects de la civilisation chinoise et qui la domine dans sa totalité ». Le 4 décembre 1975, la veille de la leçon inaugurale de son proche collègue et ami Jean-Pierre Vernant, et dix ans après le départ à la retraite de son maître Paul Demiéville, Jacques Gernet inaugure au Collège de France à l'âge de 53 ans la chaire Histoire sociale et intellectuelle de la Chine qu'il occupe jusqu'à son départ à la retraite en 1992. C'est encore à Demiéville que Gernet succède à l'Institut, élu à l'Académie des inscriptions et belles-lettres le 8 juin 1979, trois mois après le décès de celui dont il a toujours gardé le portrait dans son bureau du Collège.

Les témoignages qui ont afflué depuis la nouvelle de son décès soulignent pour beaucoup la diversité des travaux de Jacques Gernet, en même temps que leur profondeur. Pour citer l'un de ses proches collègues, Kristofer Schipper, directeur d'études émérite à l'EPHE : « Jacques Gernet allait toujours au fond des choses. On ne le voit jamais sacrifier à la facilité ou se réfugier dans de vagues généralisations. Excellent philologue, il se moquait cependant volontiers de cette acribie oiseuse qui constitue le bagage de tant de travaux sinologiques, pour ne se concentrer que sur la 
recherche de l'essentiel. Retenons de lui, de l'homme aussi bien que de son œuvre, un incontestable courage intellectuel. »

Jean Levi, pour sa part, observe que les travaux de Jacques Gernet ont été depuis toujours marqués au sceau d'une triple unité : de conviction, de méthode et de problématique. Conviction que la Chine mérite mieux qu'un intérêt de curiosité, et qu'une histoire véritablement universelle ne peut être brossée en négligeant la spécificité chinoise et ses apports aux autres cultures. Unité de méthode ensuite : il part toujours d'un trait concret, inscrit dans une réalité et un temps particuliers, pour remonter à des facteurs généraux ; en outre, il s'appuie toujours sur la comparaison pour mener ses analyses. Élevée au statut de principe heuristique, celle-ci débouche sur ce qu' on pourrait appeler une « sociologie contrastive », inspirée de Marcel Granet et de son père Louis Gernet. Unité de problématique, enfin : si les travaux de Jacques Gernet se situent explicitement dans le droit fil des enquêtes sur la longue durée et l'étude des mentalités, ils s'inscrivent aussi dans le courant de la micro-histoire.

Tout comme Jean-Pierre Vernant (1914-2007), Jacques Gernet aura marqué des générations de chercheurs, en France et dans le monde, par son enseignement, ses travaux, mais aussi son aura et sa personnalité, son élégance intellectuelle, son style d'écriture à l'inimitable « ligne claire », son engagement pour la cause rationaliste, son soutien sans faille à des chercheurs en difficulté et les encouragements qu'il n'hésitait pas à prodiguer aux plus jeunes et aux plus modestes d'entre nous. Pour moi comme pour tant d'autres, Jacques Gernet a été un maître que j'ai d'abord approché avec crainte et tremblement alors que je n'étais qu'une petite étudiante encore en quête d'un sujet de recherche. J'ai néanmoins suivi ses cours au Collège de France dans les années 1970-1980, en étant à mille lieues de me douter que je me retrouverais à cette redoutable place quelque quarante ans plus tard. Le Collège de France était alors bien différent de ce qu'il est devenu aujourd'hui. Les cours de Jacques Gernet se tenaient dans une salle sans prétention, devant un public peu nombreux, mais fidèle. Je me souviens de l'y avoir entendu parler, entre maints autres sujets passionnants, des idées dites «néo-confucéennes» sur l'éducation du fotus que j'ai bien essayé d'expérimenter plus tard sur mes deux filles - sans grand succès.

Sous ses dehors quelque peu réservés et austères, Jacques Gernet était d'une grande humanité et d'une étonnante simplicité, aimant jouer de manière impromptue du violoncelle avec des amis ou des étudiants, comme en atteste Pierre-Étienne Will, et partager des repas où l'on discutait ferme, mais qui se terminaient toujours sur un bon dessert (assez souvent un baba au rhum qui n'était jamais assez imbibé à son goût et qui recevait immanquablement une rasade d'alcool supplémentaire). Jacques Gernet aimait de cette façon mettre à l'épreuve ses idées qu'il était prêt à voir contestées, même par des juniors comme moi, mais aussi à défendre pied à pied. C'est ainsi que j'ai eu l'immense privilège d'apprendre d'un maître qui jamais ne vous opposait l'argument d'autorité, mais dont on sentait qu'il était constamment à la poursuite d'une grande idée.

Ce qui me fait conclure sur cette phrase extraite d'un roman posthume de Balzac, Les Petits Bourgeois, que Pierre-Étienne Will se rappelle avoir entendu citer par Jacques Gernet : «Le cachet du génie est une certaine apparence de facilité. Son œuvre doit paraître, en un mot, ordinaire au premier aspect, tant elle est toujours naturelle, même dans les sujets les plus élevés. » Tel était le maître que nous avons perdu.

Pr Anne CHENG, 24 juin 2018 